\title{
Contents, Vol. 109, 1945
}

\section{Index}

Amiga, H., Une modalité de fixation du greffon dans Гopé-

ration de greffe cornéenne 269

Babel, J'., Le sort des greffons cornéens transplantés . . 1

vide Franceschetti, A.

et Valerio, M., Névrome d'amputation de Torbite . 317 Bangerter, A., Zur Diagnose,

Differentialdiagnose und The-

rapie des eystoiden Maculaǘdems (Maculacysten) . . 102 Blum, J.-I)., Relations entre les

dégénérescences hérédo-fa-

miliales et les opacités congénitales de la cornée (etude

clinique et généalogique) 123

Bruckner, R., t $\tau$ ber Atmung und anaerobe Glykolyse in Ge-

weben der Rinderuvea19

Über einen Fall von traumatisch entstandener Zen-tralvenenthrombo.se 203

und Field, N. H., Beitrag zum Symptomenkomplex der ChorioreíinUis centralis serosa

(Kitahara). (Hierzu Ta-

feln III-V) 281

Coppez, L., L'électrode pyromótrique dans Toperation anti-

glaucomateuse de Vogt (Cyclodiathermoponctures) . 80 Dobrzynski, N., Nouvelle méthode

d'ionothérapie et de bal-

néothérapie oculaires ; nouvel instrument : Гécarteur-

releveur palpébral 259

Field, N. H., vide Bruckner, R. :

Franceschetti, A., et Babel, J., Essai de classification anatomique des dégénérescences familiales de la cornée.

(Avec Planches I et II) 169

Goldmann, H., Grundlagen exakter Perimetrie .... 57

- $\quad$ Ein selbstregistrierendes Projektionskugelperimeter . 71Klingler, M., L'examen oculaire des traumatisés du crane 236Louis-Bar, I)., et Pirot, G., Sur une paraplégie spasmodiqiie avec dégénérescence maculaire chez deux frères . . 32 Mihâlyhegyi, G., Fremdkörperverletzung der Linse . . 159

- $\quad$ Ein neuer Beitrag zur Entstehung des bulbären Kon-junktivalabszesses

Pavisic, Z., Die Behandlung des Ulcus serpens mit dem Sul-

fonamidpyrimidin-Präparat Debenal 227

Pirot, G., vide Louis-Bar, I). Valerio, M., vide Babel. J.

Weekers, L. et R., Les effets hypotenseurs de la diathemiiedu corps ciliaire sans perforation sclérale (cyelodia-thermie non perforante) 212

Welt, M., Etude sur les rapports entre les dimensions de lataehe aveugle de Mariotte et des angioscotomes, et latension artérielle rétinienne. (Presentation d'un campi-mètre portatif) 137 
Wölfłlin, E., Gelingt es, die angeborenen Farbensinnstòrungen zu beeinflussen? 324

ÜBERSICHTSREFERATE - REVIEWS - REVUES

ten Doesschate, G., Geschichte der Augenheilkunde. Be-

richtsjahr 1939

164

van Veelen, A. W. C., Pathologische Analomie (ausschließ-

lich Tumoren). Berichtsjahr 193843

GESELLSCHAFTSBERICHTE - SOCIETY TRANSACTIONS - SOCIÉTÉS

Croupe ophtalmologique du Lémau.

Reunion du 20 Janvier 1944 a la Clinique ophtalmologique uni-

veГsitaire de Geneve 271

Reunion des 18 et 19 mars 1944 à ГHôpital ophtalmique de Lausanne

V. Vereinigung der Basler Augenärzte. Zusammenkunft in der Universitäts-Augenklinik Basel am 24. Mai 1944 